\title{
Analysis of resting state and task-related fMRI data in small cell lung cancer patients before undertaking PCI
}

\author{
Bromis $\mathrm{K}^{1}$, Karanasiou I.S ${ }^{1}$, Matsopoulos $\mathrm{G}^{1}$, Ventouras $\mathrm{E}^{3}$, Uzunoglu $\mathrm{N}^{1}$, Karavasilis $\mathrm{E}^{2}$, Kouloulias $\mathrm{V}^{4}$, \\ Papathanasiou $\mathrm{M}^{4}$, Foteineas $\mathrm{A}^{4}$, Soldatos $\mathrm{Th}^{2}$, Iosif $\mathrm{Ch}^{2}$, Efstathopoulos $\mathrm{E}^{4}$, Kelekis $\mathrm{N}^{4}$, Kelekis $\mathrm{D}^{2}$. \\ 1. National Technical University of Athens School of Electrical \& Computer Engineering Microwave \& Fiber Optics Laboratory. \\ 2. Research Centre of Radiology and Imaging, "Evgenidion” General Hospital.
}

3 Department of Medical Instrumentation Technology, Technological Educational Institution of Athens.

4 National and Kapodistrian University of Athens, Division of Radiology-Radiotherapy II

\begin{abstract}
As the use of prophylactic cranial irradiation (PCI) increases life expectancy in certain types of cancer, there is evidence that this technique may contribute to neurocognitive deficits. The aim of this research is to investigate functional connectivity in patients before and after being administered brain radiotherapy as well as their neurocognitive functionality, depending on the course of disease and the phase of treatment and time of clinical examination. In the current study we attempt to identify potential alterations in brain function during resting state and task-related functional magnetic resonance imaging (fMRI) in small cell lung cancer patients (SCLC) patients before PCI compared to healthy subjects. The brain regions that were activated for both groups during a sequential tapping task are consistent with previous studies. However, the patient group showed a lower level of activation in the primary somatosensory cortex bilaterally. During rest, the Default Mode Network (DMN) was identified in both groups. The results presented are subject to further investigation with larger patient and control group.
\end{abstract}

Keywords- fMRI; PCI; resting state fMRI; task-related fMRI; small cell lung cancer; default mode network.

\section{INTRODUCTION}

Prophylactic cranial irradiation (PCI) is a standard technique for systemic treatment for cancer patients suffering from limited and extensive stage small cell lung cancer (SCLC) [1]. As the mean overall survival increases, the potential effect of this technique on neurocognitive impairments is becoming more clear [2]. Despite the fact that this treatment technique results in increased life expectancy, there are many cases that its use is not recommended as it has negative effects on several cognitive functions [3].

One of the most popular current methods to study neurocognitive impairments is functional Magnetic Resonance Imaging (fMRI). fMRI is a technique that uses magnetic

Manuscript received July 29, 2014. K. Bromis, I.S. Karanasiou, G. Matsopoulos and N. Uzunoglu are with the School of Electrical and Computer Eng., National Technical University of Athens, 9 Iroon Polytecnhiou, 15780, Zografos Campus (phone:+30-210-7722289; fax: +30-210-7723557; e-mail: ikaran@esd.ece.ntua.gr).

E. Ventouras is with the Department of Biomedical Technology Engineering, Technological Educational Institution of Athens, Athens, 12210 Greece (e-mail: ericvent@ teiath.gr).

G.D. Mitsis is with the Department of Electrical and Computer Engineering, University of Cyprus, Nicosia 1678, Cyprus. resonance imaging to measure brain activity by measuring changes in the local oxygenation of blood. During rest, multiple cortical brain regions are functionally linked forming resting-state networks [4]. This finding led to the hypothesis that these regions constitute a network supporting a default mode of brain function [5].

Several studies have pointed the significance of the feasibility of using resting state networks and functional connectivity for the characterization of the complex patterns of neural and behavioral consequences of cancer, treatment and brain injury [6], [7], [8].

For this reason in a recent work [9], we presented our initial results by exploring brain functional connectivity in a small sample of patients with SCLC as well as neurocognitive functionality before undertaking PCI. In the present study, having a larger sample size and more statistical power, we further elaborate on our study investigating potential differences in brain function during resting state and taskrelated fMRI in SCLC patients before PCI compared to the healthy group.

\section{METHODS}

\section{A. Acquisition}

MRI acquisition. Whole brain MRI and fMRI data were collected on a Philips 3.0T scanner (Achieva; Philips, Best, The Netherlands) at the Radiology Research Unity, Medical Imaging Department, Evgenidion Hospital, National and Kapodistrian University, Athens, Greece using an 8-channel SENSE head coil. Foam pads and headphones were used to reduce head motion and scanning noise.

Volumetric sequences. Anatomical imaging was performed with T1-weighted 3D sagittal acquisition (1.0-mm-thick slices, $0 \mathrm{~mm}$ slice gap, $\mathrm{TE}=4.6 \mathrm{msec} / \mathrm{TR}=15 \mathrm{msec}, \mathrm{FOV}=256$, and $1.0 \times 1.0 \times 1.0 \mathrm{~mm}^{3}$ reconstructed voxel size, and T2 Fluid Attenuated Inversion Recovery (FLAIR) acquisition (1.0-mmthick slices, $0 \mathrm{~mm}$ slice gap, $\mathrm{TE}=4.6 \mathrm{msec} / \mathrm{TR}=15 \mathrm{msec}$, $\mathrm{FOV}=256$, and a $1.0 \times 1.0 \times 1.0 \mathrm{~mm}^{3}$ reconstructed voxel size. 
Rs-fMRI sequence. Functional MRI data were acquired while subjects were lying quietly in the scanner with eyes closed. Whole brain rs-fMRI was performed using a gradient echo planar imaging sequence $(\mathrm{TR}=2000 \mathrm{msec} / \mathrm{TE}=30$ msec, flip angle $=90$ ). For maximum consistency, all subjects were instructed to close their eyes throughout the rs-fMRI sequence, relax, but to remain awake and motionless as much as possible during the data acquisition.

The finger tapping task consisted a block design with two conditions, sequential tapping and rest. Participants were instructed to tap the fingers of their right hand sequentially at a standard pace, starting with the thumb and finishing with the little finger. During the rest condition they were instructed to keep their eyes closed and stay calm. Each block had duration of $30 \mathrm{~s}$ and a $2 \mathrm{~s}$ verbal prompt at the beginning with the words tap and rest respectively. Each run included four repetitions of each tapping condition and five repetitions of the rest condition. The same design was repeated for the left hand for each participant.

\section{B. Participants}

Twenty two (thirteen male, nine female, age range 45-65 years) participants were included in the analysis. The sample comprised of 11 SCLC patients before PCI treatment and 11 healthy controls. All participants were native Greek speakers, right-handed (self reported), met the standard MRI safety criteria and had no history of diagnosed neurological disorder, major psychiatric disorder or treatment with psychotropic medication, including substance misuse. All participants provided written informed consent and the study was approved by the appropriate research ethics committee.

\section{Preprocessing and data analysis}

\section{Task-related fMRI data}

The preprocessing steps and the analysis were carried out with FEAT (FMRI Expert Analysis Tool, v. 5.63), which is a tool of FSL (FMRIB's Software Library, version 5.0; www.fMRIb.ox.ac.uk/fsl). Functional data were realigned to correct for motion (MCFLIRT) [10]. Non-brain structures were extracted using BET [11]. A high-pass filtering was implemented to remove low-frequency drifts (60 s cutoff) and images were spatially smoothed using a Gaussian kernel of 6 $\mathrm{mm}$ full width at half maximum and grand-mean scaled to ensure that the comparison between groups will be carried out properly. Additionally, in order to gain insight to unexpected artefacts (physiological noise due to participant motion, respiration and heartbeat, scanner drift), MELODIC (Independent Component Analysis-ICA tool in FSL) was implemented and components related to those artefacts were removed, thus improving the BOLD signal. After preprocessing the median functional image was aligned to the high-resolution T1-weighted image using a rigid body transformation [10], [12] and then registered to the $\mathrm{T} 1$ MNI152 template using affine as well as non-linear transformations with a warp resolution of $10 \mathrm{~mm}$.
The fMRI data from all the participants were analyzed at first level using FILM (FMRIB's Improved Linear Model) [13]. The contrasts of interest were left-tapping vs. baseline and right-tapping vs. baseline. Following this, higher-level analysis was conducted in order to investigate for group differences and group mean using mixed effects (ME) analysis (FLAME 1 - FMRIB's Local Analysis of Mixed Effects). Two-sample unpaired t-tests were performed in order to estimate each group's cross-subject variability separately. To obtain better results of the observed differences, we kept only those voxels with significant $(\mathrm{p}<0.05)$ effect.

\section{Resting state fMRI data}

Resting state fMRI data analysis was carried out using the MELODIC FSL tool. The data were decomposed into 42 independent components based on the fact that this approximates one sixth of the number of time-points in the scans and analyzed using multi-session temporal concatenation group ICA. Model order was estimated using the Laplace approximation to the Bayesian evidence for a probabilistic principal component model.

The spatial maps (independent components) from the multisession temporal concatenation group ICA were used to generate subject-specific versions of all spatial maps, and associated timeseries, using a regression technique called dual regression [14]. First, for each subject, the group-average spatial maps were regressed onto the subject's 4D space-time dataset (spatial regression). This resulted in a set of subjectspecific timeseries, one per group-level spatial map. Next, those timeseries were regressed into the same 4D dataset (temporal regression), resulting in a subject-specific spatial correlation maps. The aim of this work was to focus on the spatial map (component) representing the default mode network (DMN) which was visually identified based on previous studies [15], [16]. After setting a specific design matrix for cross-subject modelling, nonparametric permutation tests (5000 permutations) were performed using FSL's randomize permutation-testing tool, in order to estimate the DMN spatial map of each group and test for statistically significant differences between groups in this network. Finally, to control the probability of false activations we performed a family-wise error correction (FWE) using a threshold of $\mathrm{p}<0.05$ [17].

\section{RESULTS}

\section{Task-related fMRI data}

As we expected, for the healthy group the areas of the brain significantly activated during the left tapping task, were the premotor cortex, the primary somatosensory cortex, the primary motor cortex, the corticospinal tract and the left cerebellum. For the patient group, we observed activations in the primary somatosensory cortex, the corticospinal tract and in a smaller part of the primary motor cortex as well as the premotor cortex (Fig. 1). In addition, when comparing the two groups, the controls showed a significant higher level of activation in the primary somatosensory cortex (Fig. 2). All these activations were placed at the right hemisphere of the brain. 


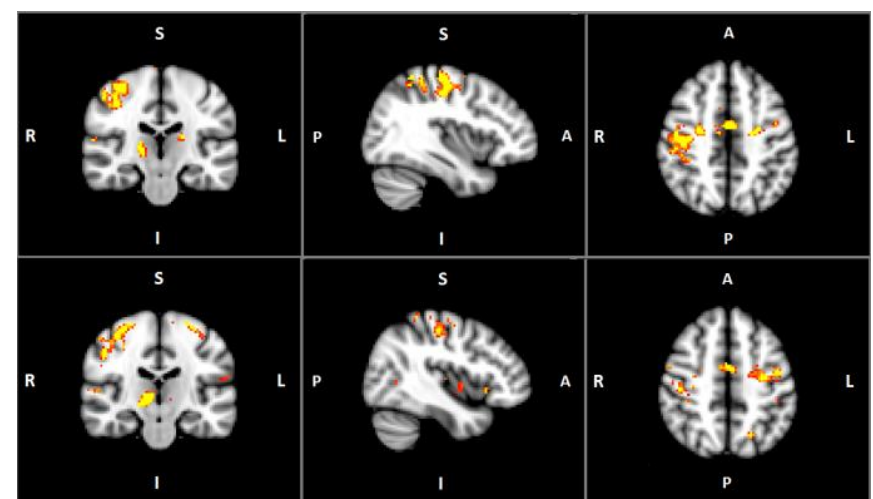

Fig. 1. Group activations in healthy subjects (top images) and SCLC patients (bottom images) for the left tapping task.

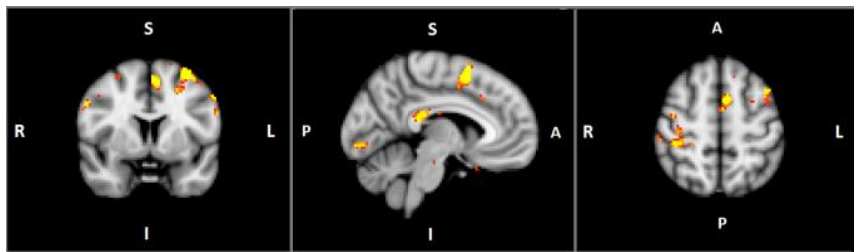

Fig. 2. Comparison between the control and patient groups for the left tapping task.

The right tapping task for the control group exhibited left hemisphere activations in the primary somatosensory cortex, the primary motor cortex, the corticospinal tract, the premotor cortex and the right cerebellum. In the patient group the activations were focused in the same brain regions as the controls except of the right cerebellum (Fig. 3). When comparing the two groups, the healthy controls showed a significant higher level of activation in the left primary somatosensory cortex (Fig. 4).

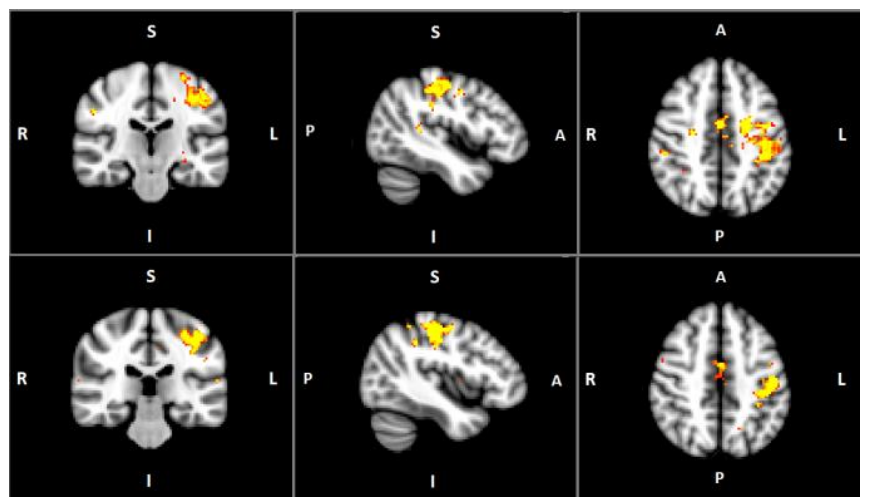

Fig. 3. Group activations in healthy subjects (top images) and SCLC patient (bottom images) for the right tapping task

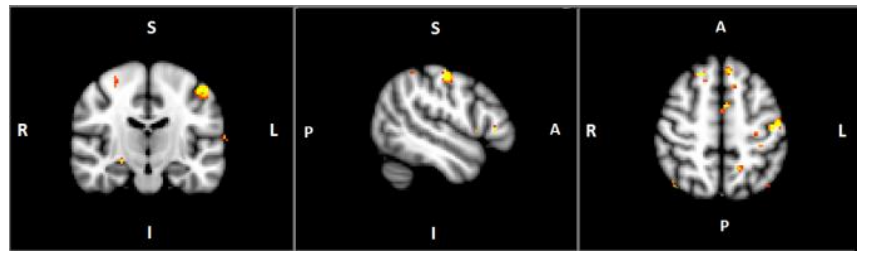

Fig. 4. Comparison between the control and patient groups for the right tapping task.

\section{Resting state fMRI data}

For both groups the DMN which includes the medial prefrontal cortex (MPFC), the medial temporal lobes (MTLs) and the posterior cingulate cortex (PCC) was significantly activated (Fig. 5).

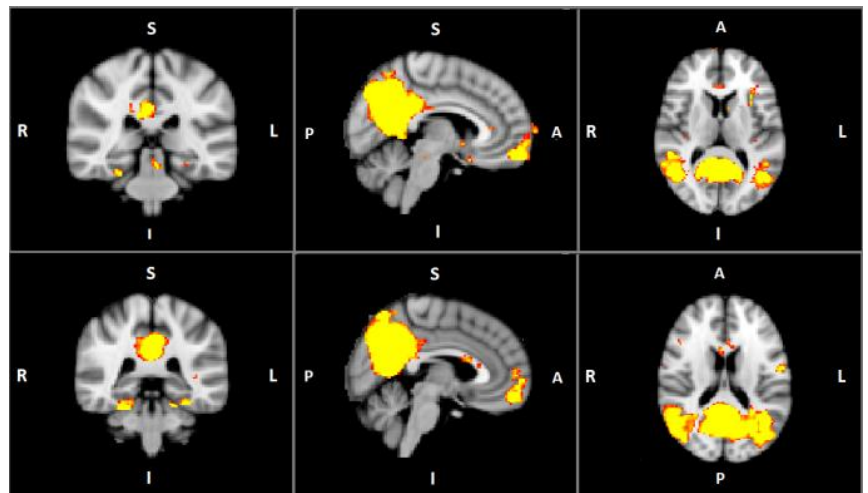

Fig. 5. Default Mode Network in healthy group (top images) and patient group (bottom images).

\section{IV.DISCUSSION AND CONCLUSION}

The goal of the current work was to investigate the brain areas that are active during a sequential tapping task in both groups, patient and healthy and see if there were any brain activity alterations in the SCLC group before undertaking PCI. Moreover, we aimed to identify the default mode network in both groups and explore for any potential alterations by comparing the two distinct groups. As with prior studies of simple motor paradigms and mainly our previous results [9], the primary motor network, including primary motor cortex, premotor cortex, primary somatosensory cortex and corticospinal tract (which originate from the neurons of the primary motor cortex and conduct motor impulses from the brain to the spinal cord) were bilaterally activated in healthy subjects during the tapping task. Similar activations were reported in the patient group. However, when comparing the two groups, the control group showed a significant higher level of activation in the left and right primary somatosensory cortex, a finding that needs further investigation as the number of participants becomes larger. During rsfMRI, the DMN was identified in both the control and patient group. This analysis is on-going and as the number of the participants is currently increased, this will ensure improved robustness and potential verification of the aforementioned preliminary findings.

In the near future, our aim is to investigate brain areas which are functionally and anatomically connected by looking at the various stages of treatment and disease. Specifically, data from patients right after PCI will be compared with those before as well as with controls, thus exploring for potential brain functional differences that may be originate from the brain radiotherapy itself. This analysis will be carried out in cancer patients for the first time to our best knowledge. 


\section{ACKNOWLEDGMENT}

This research has been co-financed by the European Union (European Social Fund - ESF) and Greek national funds through the Operational Program "Education and Lifelong Learning" of the National Strategic Reference Framework (NSRF)-Research Funding Program: THALIS - NTUA, "Study and Analysis of Medical Data using structural and functional Magnetic Resonance Imaging procedures (MRI/DTI/fMRI): Assessment of changes induced by Brain Radiotherapy" (MIS 380151).

\section{REFERENCES}

[1] J. C. Marsh, B. T. Gielda, A. M. Herskovic and R. A. Abrams, "Cognitive Sparing during the Administration of Whole Brain Radiotherapy and Prophylactic Cranial Irradiation: Current Concepts and Approaches," J. Oncol, vol. 2010, pp. 16, Apr 7, 2010.

[2] B. J. Slotman, et al, "Prophylactic cranial irradiation in extensive disease small-cell lung cancer: short-term health-related quality of life and patient reported symptoms: results of an international Phase III randomized controlled trial by the EORTC Radiation Oncology and Lung Cancer Groups," J. Clin. Oncol., vol. 27, pp. 78-84, Jan 1, 2009.

[3] A. Kanard, S. Frytak and A. Jatoi, "Cognitive dysfunction in patients with small-cell lung cancer: incidence, causes, and suggestions on management," J. Support. Oncol., vol. 2, pp. 12732; discussion 133-5, 138-40, Mar-Apr, 2004.

[4] M. E. Raichle, et al, "A default mode of brain function," Proceedings of the National Academy of Sciences, vol. 98, pp. 676682, January 16, 2001.

[5] M. P. van den Heuvel, R. C. Mandl, R. S. Kahn and H. E. Hulshoff Pol, "Functionally linked resting-state networks reflect the underlying structural connectivity architecture of the human brain," Hum. Brain Mapp., vol. 30, pp. 3127-3141, Oct, 2009.

[6] I. Bosma, J. C. Reijneveld, M. Klein, L. Douw, B. W. van Dijk, J. J. Heimans and C. J. Stam, "Disturbed functional brain networks and neurocognitive function in low-grade glioma patients: a graph theoretical analysis of resting-state MEG," Nonlinear Biomed. Phys., vol. 3, pp. 9-4631-3-9, Aug 23, 2009.

[7] L. Douw, "Neural networks and brain tumors: the interplay between tumor, cognition, and epilepsy," Vrije Universiteit Amsterdam, 2010.

[8] S. Deprez, et al., "Chemotherapy-induced structural changes in cerebral white matter and its correlation with impaired cognitive functioning in breast cancer patients," Hum. Brain Mapp., vol. 32, pp. 480-493, Mar, 2011.

[9] Bromis, K., et al., "Resting state and task related fMRI in small cell lung cancer patients", "13th IEEE BIBE 2013", art. no. 6701662, 2013.

[10] M. Jenkinson, P. Bannister, M. Brady and S. Smith, "Improved optimization for the robust and accurate linear registration and motion correction of brain images," Neuroimage, vol. 17, pp. 825841, Oct, 2002.

[11] S. M. Smith, et al., "Advances in functional and structural MR image analysis and implementation as FSL," Neuroimage, vol. 23 Suppl 1, pp. S208-19, 2004.

[12] M. Jenkinson, C. F. Beckmann, T. E. Behrens, M. W. Woolrich and S. M. Smith, "Fsl," Neuroimage, vol. 62, pp. 782-790, Aug 15, 2012.

[13] J. S. Damoiseaux, S. A. Rombouts, F. Barkhof, P. Scheltens, C. J. Stam, S. M. Smith and C. F. Beckmann, "Consistent resting-state networks across healthy subjects," Proc. Natl. Acad. Sci. U. S. A., vol. 103, pp. 13848-13853, Sep 12, 2006.

[14] N. Filippini, et al, "Distinct patterns of brain activity in young carriers of the APOE-epsilon4 allele," Proc. Natl. Acad. Sci. U. S. A., vol. 106, pp. 7209-7214, Apr 28, 2009.
[15] M. W. Woolrich, et al., "Bayesian analysis of neuroimaging data in FSL," Neuroimage, vol. 45, pp. S173-86, Mar, 2009.

[16] C. F. Beckmann, M. DeLuca, J. T. Devlin and S. M. Smith, "Investigations into resting-state connectivity using independent component analysis," Philos. Trans. R. Soc. Lond. B. Biol. Sci., vol. 360, pp. 1001-1013, May 29, 2005.

[17] S. M. Smith and T. E. Nichols, "Threshold-free cluster enhancement: addressing problems of smoothing, threshold dependence and localisation in cluster inference," Neuroimage, vol. 44, pp. 83-98, Jan 1, 2009. 